\title{
Integration of vestibular and proprioceptive signals for spatial updating
}

\author{
Ilja Frissen $\cdot$ Jennifer L. Campos $\cdot$ Jan L. Souman • \\ Marc O. Ernst
}

Received: 11 January 2011/Accepted: 25 April 2011/Published online: 18 May 2011

(C) Springer-Verlag 2011

\begin{abstract}
Spatial updating during self-motion typically involves the appropriate integration of both visual and nonvisual cues, including vestibular and proprioceptive information. Here, we investigated how human observers combine these two non-visual cues during full-stride curvilinear walking. To obtain a continuous, real-time estimate of perceived position, observers were asked to continuously point toward a previously viewed target in the absence of vision. They did so while moving on a large circular treadmill under various movement conditions. Two conditions were designed to evaluate spatial updating when information was largely limited to either proprioceptive
\end{abstract}

I. Frissen $(\bowtie)$

Institut de Recherche en Communication et Cybernétique de

Nantes (IRCCyN), UMR CNRS 6597, 1, rue de la Noë,

B. P. 92101, 44321 Nantes Cedex 03, France

e-mail: ilja.frissen@irccyn.ec-nantes.fr

I. Frissen · J. L. Souman · M. O. Ernst

Max Planck Institute for Biological Cybernetics,

Multisensory Perception and Action Group, Tübingen, Germany

\section{J. L. Campos}

Max Planck Institute for Biological Cybernetics,

Human Perception, Cognition, and Action Group,

Tübingen, Germany

\section{J. L. Campos}

Department of Psychology, University of Toronto,

Toronto, ON, Canada

\section{J. L. Campos ( $\bowtie)$}

Toronto Rehabilitation Institute, 550 University Avenue,

Toronto, ON M5G 2A2, Canada

e-mail: campos.jennifer@torontorehab.on.ca

M. O. Ernst

Department of Cognitive Neuroscience,

University of Bielefeld, Bielefeld, Germany information (walking in place) or vestibular information (passive movement). A third condition evaluated updating when both sources of information were available (walking through space) and were either congruent or in conflict. During both the passive movement condition and while walking through space, the pattern of pointing behavior demonstrated evidence of accurate egocentric updating. In contrast, when walking in place, perceived self-motion was underestimated and participants always adjusted the pointer at a constant rate, irrespective of changes in the rate at which the participant moved relative to the target. The results are discussed in relation to the maximum likelihood estimation model of sensory integration. They show that when the two cues were congruent, estimates were combined, such that the variance of the adjustments was generally reduced. Results also suggest that when conflicts were introduced between the vestibular and proprioceptive cues, spatial updating was based on a weighted average of the two inputs.

Keywords Multisensory integration - Locomotion · Vestibular · Proprioceptive - Spatial updating ·

Maximum likelihood estimation

\section{Introduction}

The most natural way for humans to move through the environment is on foot. In order to accurately produce goal-directed movements during walking and to continuously update one's position in space relative to the environment, several different sensory systems are typically used. While visual information is often considered to provide critical input about position and orientation in space, non-visual sources of information such as vestibular/ 
inertial and proprioceptive signals also play a crucial role. This is clearly demonstrated by the ability to walk accurately to previously seen targets while walking blindfolded for relatively short distances (i.e., $<20 \mathrm{~m}$ ) (Thomson 1983; Elliott 1986; Steenhuis and Goodale 1988; Rieser et al. 1990; Ellard and Shaughnessy 2003; Sun et al. 2004b; Andre and Rogers 2006; Campos et al. 2010; however, see Souman et al. 2009 for errors made at much longer distances). Vestibular information ${ }^{1}$ is mainly provided by structures in the inner ear, including the semicircular canals, which detect angular accelerations, and the otoliths, which detect linear accelerations (see Angelaki and Cullen 2008; Angelaki et al. 2009, for reviews). Proprioceptive information is provided by sensory feedback from the movement of the muscles and joints (Lackner and DiZio 2005), while efference copy information provides information about the motor commands of these movements originating in the central nervous system (Sperry 1950; Von Holst and Mittelstaedt 1950).

In the current study, we address the question of how vestibular and proprioceptive information are integrated for self-motion perception during curvilinear walking. Even though a substantial collection of research has been conducted to investigate the roles of proprioceptive and vestibular information in different aspects of self-motion perception, most of these studies have attempted to isolate these cues individually as a way of evaluating whether each is sufficient and/or necessary for veridical self-motion perception. Far fewer studies have directly addressed how these two cues interact. Those studies that have looked at cue interaction typically used somewhat artificial modes of locomotion (i.e., turning around the body axis in place) and, therefore, put into question the extent to which the results can be generalized to more commonly experienced forms of over-ground walking.

\section{The multisensory nature of self-motion perception}

Studies that have investigated the role of vestibular and/or proprioceptive information in egocentric updating have done so by systematically isolating or limiting each cue independently. For instance, various tasks have been used to assess performance under conditions in which observers actively walk through space (combined proprioceptive and vestibular inputs), walk in place (e.g., on a treadmill, producing proprioceptive but no vestibular inputs about linear translation), or are passively moved through space

\footnotetext{
1 Here we assume that most of the inputs provided during passive self-motion in the current context are largely attributable to the vestibular system. However, this is not to say that other somatosensory information from the skin during accelerations, vibrations, and wind could not play a role in self-motion perception (although several of these cues were intentionally limited in the current study).
}

(vestibular inputs but no relevant proprioceptive information from the legs). Such studies have typically demonstrated that during simple, forward movement trajectories in the absence of vision, vestibular and proprioceptive information are each sufficient to estimate travelled distance (Berthoz et al. 1995; Campos and Bülthoff (in press); Israël and Berthoz 1989; Harris et al. 2000; Mittelstaedt and Mittelstaedt 2001; Loomis et al. 1992; Loomis and Philbeck 2008; Siegle et al. 2009; Sun et al. 2004a, b) and to some extent self-velocity (Berthoz et al. 1995; Campos et al. 2009; Israël and Berthoz 1989; Siegle et al. 2009). Further, vestibular information alone has been shown to be sufficient for estimating egocentric heading direction (Butler et al. 2010; Fetsch et al. 2009) and for estimating rotations around an earth-vertical axis (Becker et al. 2002; Jürgens and Becker 2006).

Other work has considered the relative contribution of each cue by comparing performance during unisensory proprioceptive and unisensory vestibular conditions for simple behavioral tasks, such as judging displacement during forward linear movements through space (Campos and Bülthoff (in press); Mittelstaedt and Mittelstaedt 2001; Marlinsky 1999) and estimating angular displacement (Bakker et al. 1999; Becker et al. 2002; Jürgens and Becker 2006). For instance, Mittelstaedt and Mittelstaedt (2001) reported that participants could accurately estimate the length of a travelled path when walking in place (proprioception) or when being passively transported (vestibular). In their study, even though both cues appeared sufficient in isolation, when both were available at the same time (i.e., during walking through space), proprioceptive information was reported to dominate vestibular information. Similarly, others have shown that for the estimation of angular displacement when stepping in place and/or being passively rotated on a rotating platform, both proprioceptive and vestibular information can be used independently (Becker et al. 2002; Jürgens and Becker 2006). Although the displacement estimates are generally slightly higher when walking in place than when rotated passively, both show equal variance (Becker et al. 2002), which is significantly reduced when both cues are combined during active turning (Becker et al. 2002; Jürgens and Becker 2006).

Comparing how an observer responds during unisensory compared to multisensory conditions does not necessarily specify how the cues interact, nor does it allow one to quantify the relative weighting of individual cues when combined. A more quantitative method of estimating the relative contributions of vestibular and proprioceptive information is to present both simultaneously, but have them provide different (i.e., conflicting) information about the extent of self-motion. This method has been used, for instance, to quantify the contributions of visual and nonvisual cues during self-motion, by manipulating the visual 
or proprioceptive/vestibular gain (Butler et al. 2010; Campos et al. 2009; Harris et al. 2000; Rieser et al. 1995; Sun et al. 2003, 2004a). Far fewer studies, however, have systematically changed the relation between vestibular and proprioceptive cues when both are available during locomotion. Bruggeman et al. (2009) introduced conflicts between proprioceptive and vestibular inputs while participants stepped around their earth-vertical body axis on a rotating platform by providing vestibular inputs that were slower than proprioceptive inputs. Specifically, participants always stepped at a rate of 10 rotations per minute (rpm) (constituting the proprioceptive input), but because the platform rotated in the opposite direction, participants were moved through space at various different rates (constituting the vestibular input). Importantly, when the proprioceptive and vestibular inputs were of different magnitudes, the perceived velocity fell somewhere between the two presented unisensory velocities, thus suggesting that multisensory integration occurred. These results suggest that the brain uses a weighted average of vestibular and proprioceptive information (see also Becker et al. 2002).

Previous work on cue integration in self-motion perception does not clarify how vestibular and proprioceptive cues are integrated during typical walking through space. Most of the above-mentioned studies have used stepping in place to study cue integration. However, stepping in place on a rotating platform results in biomechanics that are different from over-ground walking. Because step length has been shown to be an important component when estimating walking speed and is typically a consistent metric (Durgin et al. 2009), not allowing full stride lengths to occur may inadvertently change the reliability of proprioceptive information as a cue to updating. Therefore, in the current study we used more natural, full-stride walking through space.

Research on cue integration has provided support for the idea that, for a number of different sensory systems (e.g., visual-auditory, visual-haptic), cues are often combined in a "statistically optimal" manner (e.g., Alais and Burr 2004; Bülthoff and Yuille 1996; Cheng et al. 2007; Ernst and Banks 2002; Ernst and Bülthoff 2004; Knill and Saunders 2003; Körding and Wolpert 2004; MacNeilage et al. 2007). In this context, optimality refers to a cue combination, which results in the most reliable estimate possible given the available sensory input. The model that currently best describes these results, better known as the maximum likelihood estimation (MLE) model of sensory integration, specifies three general characteristics. First, information from two or more modalities is combined using a weighted average. Second, the corresponding weights are based on the relative reliability of the unisensory cues (i.e., the inverse of their variances). Specifically, the cue with the lowest unimodal variance will be weighted highest when the cues are combined. Third, as a consequence of integration, the variance observed in multimodal conditions will be lower than that observed in either of the unimodal conditions alone.

Attempting to study cue integration during self-motion, however, presents unique challenges because there is a very tight coupling between vestibular and proprioceptive information during walking [see also Campos and Bülthoff (in press)]. This makes it extremely difficult to obtain independent unisensory estimates. While it is relatively trivial to isolate exteroceptive signals such as visual, auditory, and haptic inputs, it is nearly impossible to completely "turn off" the vestibular system or the proprioceptive system for healthy individuals. Consequently, while walking in place on a treadmill, the information from the proprioceptive system is generally consistent with movements associated with walking through space, whereas the vestibular system specifies a stationary position, thus creating a potential conflict. The reverse conflict occurs when one is moved passively through space such that vestibular input specifies movement through space, while the proprioceptive input from the legs specifies a stationary position. That said, these two conflicts are not necessarily complementary in nature. Specifically, there are numerous instances where vestibular excitation is experienced without contingent proprioceptive information from the legs, including whenever we move our head or when moving in a vehicle. However, under normal circumstances there can be no proprioceptive activity consistent with translational walking behaviors, without experiencing concurrent vestibular excitation.

With this in mind, the aforementioned "unisensory" proprioceptive or vestibular conditions can, in fact, be considered multisensory conditions in which the two systems specify extreme conflicting values (movement vs. no movement). ${ }^{2}$ In particular, for the unisensory proprioceptive condition, it can be argued that because of the strong coupling between proprioceptive and vestibular signals under natural walking conditions, the brain has adopted a strategy of always integrating the two signals (irrespective of the size of the conflict). If this conjecture is valid, it presents a serious problem for assessing the contributions of proprioceptive information to self-motion perception using traditional methods, as it effectively cannot be isolated in healthy adults. This would of course also affect conclusions from previous research relying on the "walking in place" condition. To compound the problem, these tasks often require participants to use the information from their legs to imagine moving through space (Becker et al. 2002; Jürgens and Becker 2006; Mittelstaedt and

\footnotetext{
2 Note that despite the fact that the term "unisensory" may not be completely appropriate in this context, we will nonetheless use this terminology throughout the paper for simplicity.
} 
Mittelstaedt 2001). This introduces additional cognitive variables that can potentially create additional biases in this condition. Conversely, these considerations do not hold as strongly for the unisensory vestibular condition as the coupling is such that it does not necessarily warrant a strategy of mandatory integration.

\section{Current study}

The present study has two aims. First, we wanted to study cue integration during full-stride, curvilinear walking by introducing subtle conflicts between the vestibular and proprioceptive cues. Second, our goal was to gain insight into the processes involved in the unisensory conditions as used in previous research. In order to achieve this, we measured self-motion perception during multisensory conditions (walking through space; WTS) and during the two unisensory conditions, including a passive movement condition (PM) and a walking in place condition (WIP).

For this, we used a large circular treadmill, which featured a motorized handlebar that could move independently of the treadmill disk. This unique instrument allowed us to manipulate the two signals independently during walking. We evaluated spatial updating using a continuous pointing task similar to that introduced by Campos et al. (2009) and Siegle et al. (2009), which expanded upon a paradigm originally developed by Loomis and et al. (Fukusima et al. 1997; Loomis et al. 1992; Loomis and Philbeck 2008). This task involves continuous pointing to a previously viewed target during self-motion in the absence of vision. This method provides continuous information about perceived target-relative location and thus about self-velocity during the entire movement trajectory. It also maintains advantages over other methods that rely on the implicit assumption that participants have an accurate concept of units of measurements in degrees. For instance, some tasks require participants to use a button press or verbal response after rotating a certain number of degrees (Becker et al. 2002; Bles 1981; Bles and de Wit 1978; Bruggeman et al. 2009; Jürgens and Becker 2006; Marlinsky 1999). This is not as natural or intuitive as goal-directed target updating and requires additional transformations. Further, unlike other tasks such as those requiring the active reproduction of a previously experienced path (Berthoz et al. 1995; Glasauer et al. 1994, 2002; Israël et al. 1997; Marlinsky 1999), this updating task occurs online in real time and therefore avoids the introduction of potential memory-related effects associated with comparing two sequentially presented movements.

Based on the three tenets of the MLE model described earlier, it is expected that the multisensory condition (WTS) will show evidence of a weighted average of proprioceptive and vestibular signals. In particular, the MLE model predicts that spatial updating performance in the multisensory condition will fall between that in the two unisensory conditions when the two signals present conflicting information. Also, the variance in the multisensory condition estimates (without conflict) should be less than that in either of the unisensory conditions. By comparing the pattern of pointing responses in the PM condition with that in the WTS condition (without conflict), we can evaluate whether the capacity for spatial updating (i.e., target relative position and egocentric velocity) is different when predominantly vestibular inputs are available, compared to when both proprioceptive and vestibular inputs are available. The WIP condition will allow us to evaluate whether imagining one's movement through space using proprioceptive information obtained through stepping in place is sufficient for spatial updating. Because there are characteristic patterns of pointing movements observed during actual self-motion perception through space, these can be compared to the patterns of responding during imagined self-motion through space. If the patterns of pointing during the WIP condition demonstrate clear evidence of perceived spatial updating, this could validate the use of this task in studying the contributions of proprioceptive information to curvilinear self-motion perception. If, however, the pattern of pointing deviates substantially from that observed during actual movement through space, this would reveal important characteristics about spatial updating in the absence of physical movement. Because walking/stepping in place paradigms have been frequently adopted to study the influence of different sensory information on various aspects of self-motion perception, these results have wide-ranging implications.

\section{Methods}

\section{Participants}

Thirteen participants (seven women) between the ages of 18 and 35 years, with normal or corrected-to-normal vision, and no known vestibular or motor dysfunctions, volunteered for this study. The first two authors were among the participants. Two other participants had completed a similar pilot study but had not been debriefed about its purpose or manipulations. Thus, with the exception of the authors, all were naïve to the purpose of the experiment. Participants were paid eight Euros per hour of participation. The experiment was conducted in accordance with the ethical standards specified by the 1964 Declaration of Helsinki.

Experimental setup

The experiment was conducted using the circular treadmill (CTM) setup available at the Max Planck Institute for 


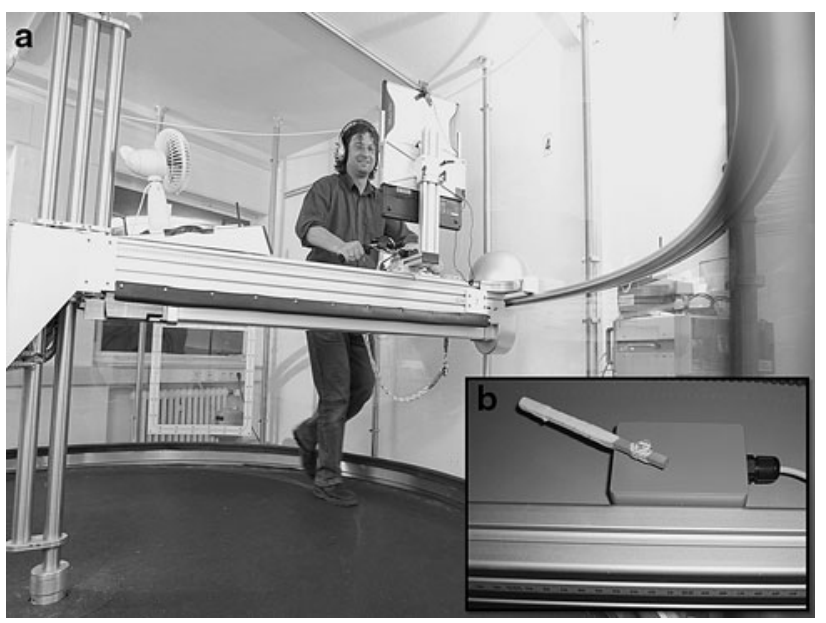

Fig. 1 The experimental setup. a The circular treadmill (CTM) at the Max Planck Institute for Biological Cybernetics. (photograph courtesy of Axel Griesch.) b The pointing device

Biological Cybernetics in Tübingen (see Fig. 1a). This treadmill consists of a large motorized wooden disk $(\varnothing=3.6 \mathrm{~m})$ covered with a slip-resistant rubber surface and a motorized handlebar. The disk and handlebar can be actuated independently from each other. The disk's maximum angular velocity is $73 \%$, and the handlebar can reach a maximum velocity of $150 \%$ s. Both disk and handlebar are equipped with an angular position encoder (resolution $0.2^{\circ}$ ). The participants held on to bicycle handlebars mounted onto the motorized handlebar at a radius of $1.28 \mathrm{~m}$. Throughout the experiment, the devices were accelerated and decelerated using a raised cosine velocity profile with peak accelerations ranging between 10 and $20 \% \mathrm{~s}^{2}$. When moving through space, the participant always moved in a clockwise direction (seen from the top). Walking on the CTM is natural and intuitive and does not require any explicit training. Unpublished observations with fourteen participants (7 women) showed that when walking in place or walking through space on the CTM, typical gait parameters such as step length $(0.5-0.7 \mathrm{~m})$, step frequency $(1.3-1.9 / \mathrm{s})$, and walk ratio (i.e., the ratio between step length and frequency; 0.34-0.37) for walking speeds between $30 \%$ and $60 \%$ s were similar to those reported for over-ground walking (e.g., Alton et al. 1998; Riley et al. 2007).

In all conditions, there was a constant sinusoidal motion superimposed on the handlebar motion (frequency $=$ $0.65 \mathrm{~Hz}$; amplitude $=5 \%$ ). Its purpose was to reduce the reliability of any motion cues acquired through the handlebar's pull on the arms, as these cues normally are not available during walking. Participants also wore a blindfold, earplugs, and a wireless headset, which played an acoustic noise mask. The mask was a mixture of white noise and recordings made from the moving disk played at the highest volume tolerable to the participant. Responses were collected using a custom-built pointing device (Fig. 1b) that was mounted on the handlebar within comfortable reaching distance of the right hand (at a radius of $0.93 \mathrm{~m}$ from the center of the disk). The pointing device consisted of a USB mechanical rotary encoder (Phidgets Inc.) with a pointing rod attached and encased in plastic. The encoder's resolution was 80 counts per revolution (i.e., $4.5^{\circ}$ ). For each trial, we recorded the elapsed time and the positions of the disk, handlebar, and pointer at a sampling rate of $\sim 77 \mathrm{~Hz}$. The target was located in one of the corners of the laboratory. It was clearly marked with a black cross $(20 \mathrm{~cm}$ high and wide, at the level of the pointer) taped onto the white wall (see also Fig. $2 \mathrm{a}$ for more details on the geometry of the setup).

Procedure

To measure spatial updating performance, participants engaged in a continuous pointing task. They were first shown the target positioned in the corner of the laboratory under full visual conditions. They then donned the blindfold and were instructed to continuously adjust the rod on the pointing device so that it was always aimed at the target during movement.

The pointing task was performed under four different movement conditions, which are summarized in Table 1. There were two unisensory conditions, passive movement (PM) and walking in place (WIP). In the PM condition, participants stood still while they were passively moved by the CTM. In the WIP condition, participants walked in place on the treadmill and did not move through space. In the WIP condition, like in previous studies, participants were instructed to use the proprioceptive information from their legs to update their egocentric position as if they were moving through space at the specified velocity by the CTM. The third condition was the multisensory walking through space (WTS) condition during which both vestibular and proprioceptive systems indicated self-motion. This condition consisted of both congruent and incongruent trials. In the congruent trials, participants walked behind the handlebar while the treadmill disk remained stationary. Thus, the vestibular and proprioceptive inputs conveyed the same movement velocities; in other words, the proprioceptive-vestibular gain was 1.0. In the incongruent trials, systematic conflicts were introduced between the vestibular and proprioceptive inputs. This was achieved by having participants walk at one rate, while the disk moved at a different rate. Specifically, proprioceptive gains of 0.7 and 1.4 were applied to two vestibular velocities $(25 \%$ and $40 \%$ ) (see Table 1). To achieve a gain of 0.7 , the disk moved in the same direction as the handlebar at $30 \%$ of its speed. To achieve a gain of 1.4 , the disk moved at $40 \%$ of the handlebar speed but in the opposite direction. Finally, 

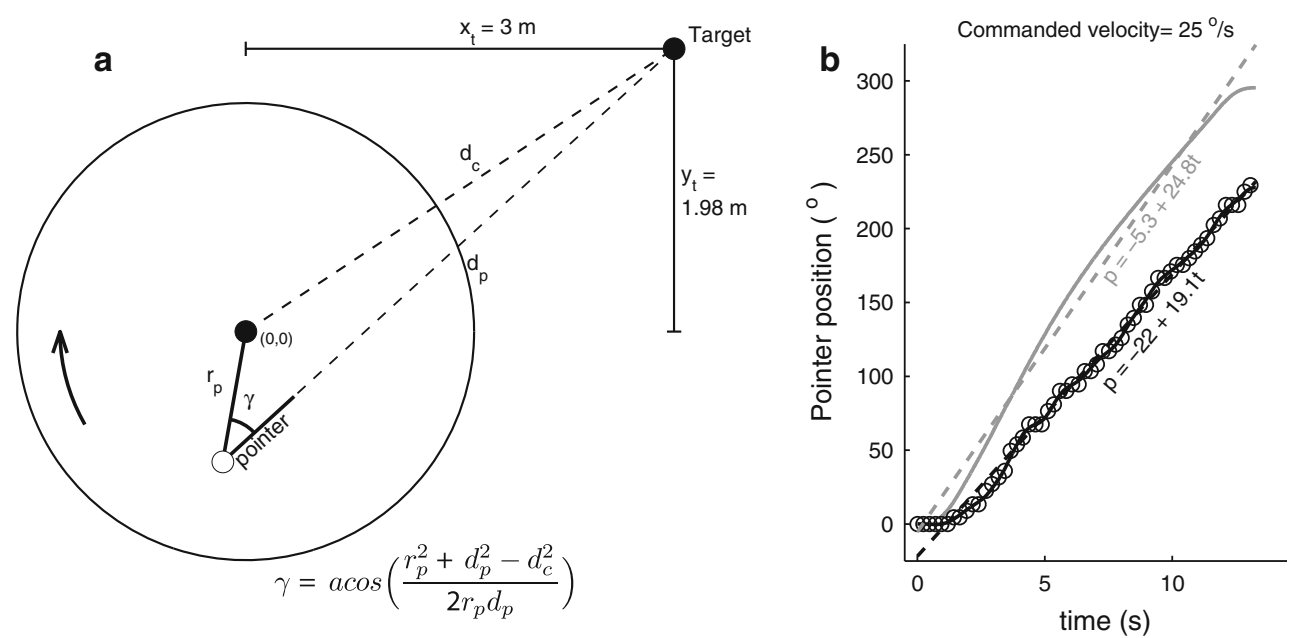

Fig. 2 Data analysis. a Geometry of the setup. The circle represents the CTM with the origin of the reference frame set in its center. The pointing device is at a distance of $r_{p}(0.925 \mathrm{~m})$ from the center. The target is in the top right corner at $\left(x_{t}, y_{t}\right)$. The distance of the target to the origin $\left(d_{c}\right)=3.6 \mathrm{~m}$. The distance of the pointer to the target $\left(d_{p}\right)$ depends on the known (i.e., measured) position of the pointer. With these values known, pointer angle $\gamma$ of the target relative to the handlebar can be calculated using the law of cosines. b Calculating the mean velocity using linear regression. The graph shows the pointer position over the course of one exemplary trial in the walking in place condition with a commanded velocity of $25 \%$ s. The black markers (here down sampled by a factor 15 for illustrative purposes)

Table 1 Overview of the different movement conditions

\begin{tabular}{|c|c|c|c|c|c|}
\hline \multirow{2}{*}{$\begin{array}{l}\text { Movement } \\
\text { conditions }\end{array}$} & & \multicolumn{2}{|c|}{ Movement velocities $(\% / \mathrm{s})$} & \multirow[t]{2}{*}{ Gain } & \multirow{2}{*}{$\begin{array}{l}\text { Main source } \\
\text { of sensory } \\
\text { information }\end{array}$} \\
\hline & & Vestibular & Proprioceptive & & \\
\hline Passive movement & PM & $\begin{array}{l}15,20,25, \\
30,35,40\end{array}$ & 0 & N/A & Vestibular \\
\hline Walking in place & WIP & 0 & $\begin{array}{l}17.8,25.0 \\
28.4,35.0 \\
40.0,56.0\end{array}$ & N/A & Proprioceptive \\
\hline \multirow{6}{*}{$\begin{array}{l}\text { Walking through } \\
\text { space }\end{array}$} & \multirow[t]{6}{*}{ WTS } & \multirow[t]{3}{*}{25} & 17.8 & 0.7 & Vestibular \\
\hline & & & 25.0 & 1.0 & \multirow[t]{5}{*}{ Proprioceptive } \\
\hline & & & 35.0 & 1.4 & \\
\hline & & \multirow[t]{3}{*}{40} & 28.4 & 0.7 & \\
\hline & & & 40.0 & 1.0 & \\
\hline & & & 56.0 & 1.4 & \\
\hline \multirow[t]{2}{*}{ Full-cue walking } & \multirow[t]{2}{*}{ ALL } & \multirow{2}{*}{$\begin{array}{l}15,20,25, \\
30,35,40\end{array}$} & $15,20,25$ & \multirow[t]{2}{*}{1.0} & Vestibular \\
\hline & & & $30,35,40$ & & Proprioceptive \\
\hline
\end{tabular}

the Full Cue Walking (ALL) condition was identical to the congruent WTS condition except that participants walked with their eyes open and thus had full vision during the entire movement. This condition served as a control condition and was not meant to measure spatial updating per se, but rather to assess participants' abilities to adjust the pointer appropriately when moving. are the actual recordings made from the rotary encoder. The smooth black line shows the same data but low pass filtered at $1 \mathrm{~Hz}$. The black dotted line shows the linear regression of the filtered data. The parts of the profile that corresponded to the acceleration and deceleration phase of the CTM were not used in the regression analysis. The gray lines represent the ideal case for the same trial that was calculated based on the trigonometry illustrated in panel a. The meandering nature of the ideal profile reflects the changes in updating velocity inherent to the eccentric rotation with respect to a fixed point in space (see also "Results"). The slope of the regression line (text inserts) was taken as the estimate for the mean pointing velocity, the intercept was ignored

The experiment was run in two sessions of approximately $1 \mathrm{~h}$ each, separated by at least 1 day. Each condition included six movement velocities (see Table 1), which were all tested six times for a total of 36 trials per condition, equally distributed across the two sessions. Between conditions there were short mandatory breaks, and participants were allowed to take as many breaks as they needed for as long as they needed. Conditions were blocked and counterbalanced, apart from the ALL condition, which was always completed first. This was done to provide participants with the opportunity to practice the task, to calibrate to the dimensions of the room and to become familiar and comfortable with moving on the CTM and using the pointer. In the first session, participants received three additional practice trials at the start of each condition (only congruent trials in the WTS condition), in order to learn the procedure and become comfortable with the different movement conditions.

Each trial consisted of two phases, a repositioning phase and the experimental pointing task phase. In the repositioning phase between trials, the participant was moved to a new random location in space while wearing the blindfold in order to ensure different starting positions in each trial and to limit visual feedback on performance on the previous trial. This movement was preceded by a single beep over the headphones to forewarn participants of the impending movement. During this phase, they were moved 
through space at $20 \%$ for a random duration between 10 and $12 \mathrm{~s}$, while at the same time they walked at a velocity of $40 \%$ s. There was no task for the participant to complete other than to follow the handlebar. After reaching the new starting position (indicated by a double beep), the participant removed the blindfold, oriented him/herself, and located the target. They then adjusted the pointer so that it was aimed directly at the target. When ready, the participant then put the blindfold back on and initiated the second phase of the trial by pressing a button with the non-pointing hand.

In the experimental phase, the movement parameters were specified by the condition tested. The duration of this phase varied between $\sim 10$ and $\sim 19 \mathrm{~s}$ in each trial, with a constant velocity portion between 8 and $12 \mathrm{~s}$. The participants were instructed to point continuously toward the remembered location of the target. For the WIP condition, regardless whether participants experienced true "vection" during walking (i.e., an illusory sensation of movement through space), they were asked to use the proprioceptive information to update their target-relative position as if they were moving through space at the specified rate and to point accordingly.

\section{Data analysis}

The raw data from the pointing device provided continuous angular position information as participants were moving. Participants aligned the pointing device with the target at the beginning of each trial. This initial value was subtracted from the data in that trial so that each position profile started at zero. In other words, pointing profiles were aligned so that the target position at the start of the trial was at zero with respect to the participant (or rather, the pointing device). For each condition, the pointing profile was compared to the profiles obtained from the ALL cue condition. The raw data were analyzed in two different ways. First, to assess the instantaneous rate of change in pointing, the recorded pointing angle profiles were transformed into pointing rate profiles by differentiation with respect to time. Second, to make statistical comparisons between conditions, the mean pointing rate was calculated.

\section{Pointing rate profiles}

The purpose of calculating the pointing rate profiles was to see whether participants changed their pointing rate in accordance with the geometry of the setup (i.e., pointer direction relative to target). During accurate pointing, the pointing rate should increase when approaching the target, peak upon target passage and decrease when moving past it. The angular velocity profiles, that is, the rate at which the orientation of the pointer changed over time, were obtained by taking the first derivative of the pointer orientation. Per individual and condition, pointing rate profiles from across the different trials were averaged after first aligning them with respect to the position on the CTM and then computing the median velocity in bins of $3^{\circ}$. Finally, to obtain a group average and associated variability, the mean pointing rate and standard error across the individual profiles were computed.

\section{Mean pointing rate}

Performance on a single trial was summarized in a single value representing the mean pointing rate. This was estimated by taking the slope of a linear regression on the position profiles with respect to time (see Fig. 2b). The validity of this method was demonstrated by a very high correlation $\left(r^{2}=0.94\right)$ between the velocity commanded to the device and the ones obtained from the regression method (e.g., the gray line in Fig. 2b).

\section{Statistical analyses}

Statistical analyses were conducted using repeated measures ANOVAs with SPSS 15.0. Violations of sphericity were addressed using Huyn-Feldt correction or in extreme cases $(\varepsilon<0.5)$ by using the results from the MANOVA.

\section{Results}

\section{Pointing rate profiles}

The black lines in panels A, B, and C of Fig. 3 show the profiles for the PM, WTS, and WIP conditions, respectively. Each curve corresponds to the $25 \%$ s (bottom line) and $40 \%$ s (top line) movement velocities. As mentioned earlier, during correct spatial updating the rate at which the pointer is adjusted is not constant, but rather, changes periodically because of the changing distance between the pointer and the target as one moves on the CTM. The ALL cue condition baselines (in gray) were generally consistent with accurate performance; however, they demonstrated some deviations in rate. This was mainly due to biomechanical constraints imposed by the pointing device. At around $120^{\circ}$, participants had to readjust their grasp on the pointer. Participants compensated for the time that the pointer was at rest by introducing a brief acceleration in the adjustment of the pointer, which in fact reflects their awareness of their position in space. A similar pattern of pointing behavior was observed for the PM and the WTS (congruent) conditions. Importantly, the profiles in the WIP condition (Fig. 3c) were essentially flat and lacked any periodicity or even the brief compensatory acceleration. 


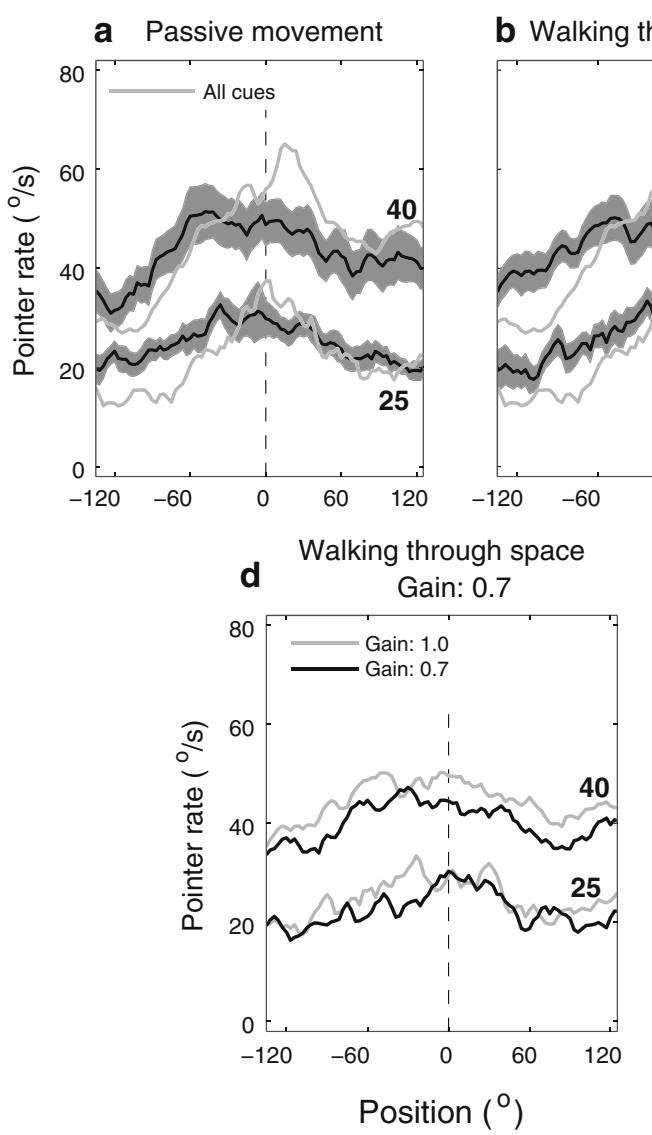

Fig. 3 Velocity profiles for the different movement conditions showing the group mean of the instantaneous rotational velocity of the pointer for the $25 \%$ and $40 \%$ s test velocities. The $x$-axis gives the position of the participant relative to the target. Because of the eccentric rotation with respect to a fixed point in space, the update rate should be periodic, with its peak around a position of zero (i.e., target passage). The black lines represent the profiles for the panel's corresponding condition. The shaded areas show the standard error of the mean. The gray lines across panels a-c are the same and represent

The subset of WTS conditions, which involved a conflict between the vestibular and proprioceptive input is plotted separately. Figure $3 \mathrm{~d}$ represents the velocity profiles when the proprioceptive input was 0.7 times that of the vestibular input and Fig. 3e represents those in which the proprioceptive input was 1.4 times that of vestibular input. Together, these reveal that the conflict led to systematic changes in the pointing rate profile. For both the $25 \% \mathrm{~s}$ and $40 \%$ vestibular velocities, we see that when the gain was 0.7 , the overall updating velocity decreased, whereas it increased when the gain was 1.4 (see below for an analysis of cue interactions using the averaged data).

\section{Cue interactions}

In order to test the MLE predictions, participants' mean pointing rates were examined. The MLE prediction specifying that estimates from two or more modalities are

\section{Walking in place}

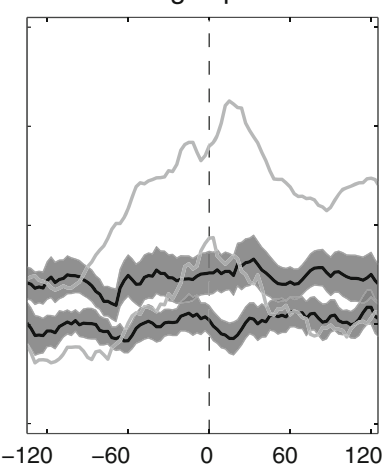

Walking through space

e Gain: 1.4

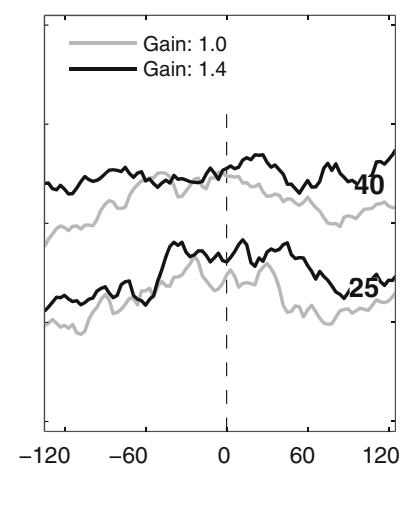

the pointer profiles for the ALL condition when walking through space at $25 \%$ (bottom line) and $40 \%$ (top line) and serve as a reference. a Passive movement. b Walking through space. c Walking in place. Note the lack of periodicity in the walking in place condition. Panels $\mathbf{d}$ and $\mathbf{e}$ highlight the conditions where there was a conflict between vestibular and proprioceptive inputs. The gray lines are for the congruent input condition (identical in both panels) and the black lines show the results for the non-unity gains

combined using a weighted average was first evaluated. The effects of the conflicts between the vestibular and proprioceptive inputs on the mean pointing rate are shown in Fig. 4a. These mean values confirmed that the pointing rate was systematically affected by the different gains for each of the two vestibular velocities tested $\left(25^{\circ} / \mathrm{s}\right.$ and $\left.40^{\circ} / \mathrm{s}\right)$, indicating that a weighted average was used. When the gain between the proprioceptive and vestibular velocity was 1.0 (i.e., no conflict), performance was close to veridical. When the gain was 0.7 , the mean pointing rate decreased and when the gain was 1.4 , the mean pointing rate increased. This was confirmed by a 2 (test velocity: $25 \%$ vs. $40 \%$ ) $\times 3$ (gain: $0.7,1.0,1.4)$ repeated measures ANOVA, which revealed a significant main effect of gain $(F(2,24)=48.26$, $P<0.0001)$ and a significant main effect of test velocity $(F(1,12)=187.92, P<0.0001)$, but no significant interaction effect $(F(2,24)=2.89, P=0.098)$. A planned comparison comparing velocity estimates for gains 0.7 and 


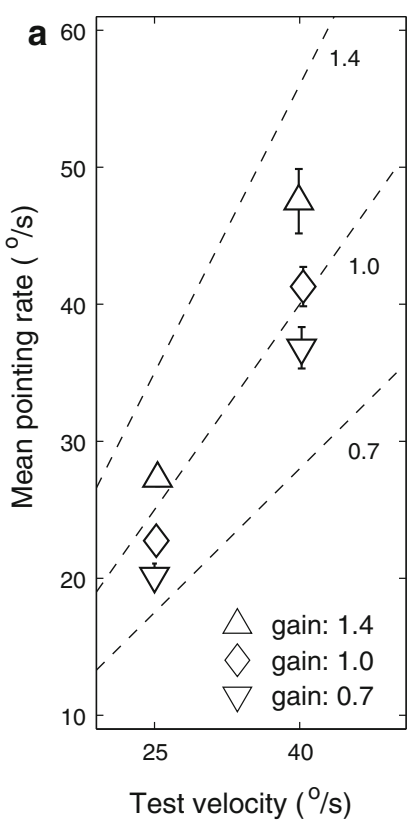

Fig. 4 Mean pointing rates. The graphs show the mean pointer velocities as a function of test velocity, both calculated using the regression method (see "Data analysis"). a Bimodal conditions with and without conflicting inputs. The diamond markers correspond to the cases where the inputs were congruent. The upward triangles show the mean pointing velocities when the proprioceptive inputs were $1.4 \times$ that of the vestibular inputs. The downward triangles show the mean pointing velocities when the proprioceptive inputs were

1.0 was significant $(F(1,12)=15.52, P<0.01)$, as was the planned comparison comparing gains 1.4 and 1.0 $(F(1,12)=46.31, P<0.0001)$. There was no significant interaction with test velocity for the former comparison, while it was close to being significant for the latter comparison $(F(1,12)=4.42, P=0.057)$, suggesting a somewhat larger effect of the gain of 1.4 for the faster test velocity than for the slower test velocity. Using the group mean pointing rate in the conflict conditions, the relative weights of vestibular and proprioceptive cues were calculated to be 0.62 and 0.38 (i.e., 1-0.62), respectively.

We next tested the MLE prediction that the variance observed in multimodal conditions (WTS) would be lower than that observed in either of the unimodal conditions alone (PM and WIP). We used the individual inter-trial standard deviation (SD) of the mean pointing rate across the six repetitions, which can be assumed to be equal to the sensory noise (Faisal and Wolpert 2009; Jacobs 1999; Nardini et al. 2008). For the WTS condition, the SD was calculated for the congruent (i.e., gain $=1.0$ ) trials only, and for both the WIP and PM conditions the SD was calculated for the trials with the same velocity values (i.e., $25 \%$ and $40 \%$ s). The SDs are summarized in Fig. 5a. On average, the bimodal condition (WTS) produced a mean SD that was smaller than either of the corresponding
$0.7 \times$ times that of the vestibular inputs. The error bars show standard errors of the mean across participants. The dashed lines labeled 1.4 and 0.7 correspond to the pointing rates that would be expected if participants were exclusively (and perfectly) using proprioceptive information. b Mean pointing velocities in the ALL cue control, unimodal, and bimodal (with congruent inputs) conditions. The diagonal line indicates accurate performance

unimodal conditions. A 3 (condition: WIP, PM, WTS) $\times 2$ (velocity) repeated measures ANOVA showed a near significant effect of condition $(F(2,24)=2.80, P=0.08)$. However, one participant produced a large variability in WTS at $40 \%$ s (participant $7, \sigma=10.1 \%$ ), which may have had a disproportional effect on the ANOVA. Indeed, after excluding this participant's results, the ANOVA revealed a significant effect of condition $(F(2,22)=3.94, P=0.034)$, and no effect of velocity $(F(1,11)=3.06, P=0.11)$. There was also no significant interaction effect $(F<1)$. Since there was no significant difference between WIP and PM either $(F(1,11)=1.89, P=0.20)$, we created a single unisensory condition by averaging across the WIP and PM conditions and compared it with the WTS condition in a 2 (condition: unisensory vs. WTS) $\times 2$ (velocity) repeated measures ANOVA. As before, there was a significant main effect of condition $(F(1,11)=8.29, p=0.015)$ but no significant effect of velocity nor and interaction (both $P$ values $>0.14$ ).

We also inspected the relationship between variances in the different conditions for individual participants. To compare the different movement conditions, we calculated the following metrics, $\mathrm{SD}_{\mathrm{WTS}} / \mathrm{SD}_{\mathrm{WIP}}$ and $\mathrm{SD}_{\mathrm{WTS}} / \mathrm{SD}_{\mathrm{PM}}$, which under the MLE model should both be less than 1 . The results are shown in Fig. 5b, separately for the two movement velocities. In this format, all data points in the 


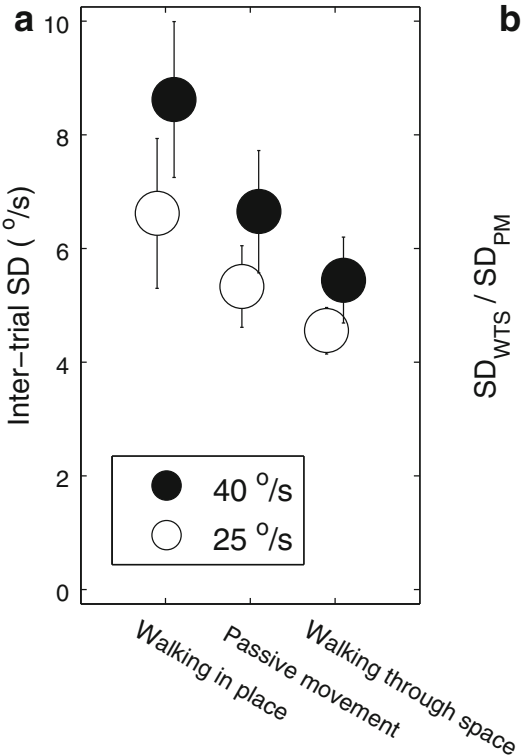

Fig. 5 Estimates of sensory noise. a The mean inter-trial standard deviation (SD) for the WIP, PM, and WTS conditions. Error bars show the standard error of the mean across participants. b Individual participant results for the two test velocities. The MLE predicts that the variance in the WTS condition should be lower than both WIP and

lower-left quadrant (gray-shaded area) are compatible with the MLE model. Four participants, including the second author, were completely compatible. Six more participants were consistent with MLE in at least one of the two test velocities.

Walking in place and passive movement

Figure $4 \mathrm{~b}$ plots the observed mean pointing rates as a function of the presented velocity for the WIP, PM, WTS (congruent), and ALL conditions. Mean pointing rates in the ALL condition (Fig. 4b, open squares) revealed very small errors across the different test velocities, ranging from $-0.04 \%$ to $-1.08 \%$ and an overall mean signed error across velocities of $-0.42 \%$. A one-way repeated measures ANOVA revealed that the overall error was significant $(F(1,12)=7.79, P=0.016)$; however, these error values did not change as a function of movement velocity $(F(5,60)=1.54, P=0.19)$.

The results from the PM condition (Fig. 4b, filled circles) demonstrated accurate performance throughout the entire range of test velocities. We calculated the error by subtracting the mean pointing rate from the presented velocity. Errors were on average $-0.78 \%$, ranging from $1.58 \%$ to $-2.31 \%$ s. A one-way repeated measures ANOVA revealed that error values did not change as a function of velocity $(F(5,60)=1.83, P=0.15)$, nor was the overall error significant $(F<1)$.
PM. Plotted are $\mathrm{SD}_{\mathrm{WTS}} / \mathrm{SD}_{\mathrm{WIP}}$ versus $\mathrm{SD}_{\mathrm{WTS}} / \mathrm{SD}_{\mathrm{PM}}$ for each participant and for each velocity. If the MLE holds, all data points should lie in the lower left (shaded) quadrant. One extreme point of participant 7 , at coordinate $(6.7,2.3)$, is not shown. The triangles and diamonds show the results for the first and second author, respectively

The results from the WIP condition (Fig. 4b, open circles) demonstrated a systematic underestimation of velocity. The curve increases monotonically, but with a shallower slope than accurate performance (gain factor 0.78). That is, the magnitude of underestimation increased with increasing velocity. The overall error was significant $(-6.28 \%$, $F(1,12)=11.70, P<0.01)$, and so was the effect of velocity (multivariate $F(5,8)=6.01, P<0.05$ ), indicating a linear increase in absolute error as velocity increased (trend analysis: $F(1,12)=5.23, P<0.05)$. Consistent with the lower slope, the error in proportion to the tested velocity was $22.5 \%(\mathrm{SE}=6.2 \%)$ on average and did not differ significantly across the various test velocities $(F<1)$.

\section{Discussion}

Cue integration during full-stride curvilinear walking

To test whether the MLE model of sensory cue integration (Ernst and Banks 2002) applies to the integration of vestibular and proprioceptive information, we evaluated participants' ability to update their target-relative position under conditions where these two inputs specified either congruent or conflicting values. Overall, the results demonstrate evidence for the integration of proprioceptive and vestibular information that is qualitatively consistent with the MLE model. This was most clearly shown by the 
effects of introducing a conflict between vestibular and proprioceptive information during curvilinear walking. The updating rates changed in a way that would be predicted given a weighted average of the two cues. As an illustration, when participants were moved through space at a rate of $40 \%$ s (vestibular cue), but walked at a rate of $56 \%$ (proprioceptive cue with a gain of 1.4 relative to vestibular cue), an average perceived rate of $48 \%$ was observed. These results are consistent with those of Bruggeman et al. (2009) who found that participants perceived themselves to be moving faster when actively stepping through space (by turning around their vertical body axis) compared to stepping in place. When participants in their study stepped at one rate $(10 \mathrm{rpm})$, but moved through space at another (e.g., $6 \mathrm{rpm}$ ), they perceived themselves as moving at approximately $7.5 \mathrm{rpm}$.

We also found evidence for a reduction in variance in the multisensory condition (WTS) compared to the unisensory conditions (PM and WIP). Whether this reduction in variance was statistically optimal could not be determined, because the unisensory proprioceptive condition (WIP) showed biased estimates. This precluded the ability to calculate independent estimates of proprioceptive and vestibular variances. This bias also made it impossible to calculate the relative weights of the two cues (as will be discussed further below). That said, given that there were no such biases in the PM condition, comparing the responses in the PM condition to those in the WTS condition is entirely valid. Notably, in the majority of cases, the WTS condition revealed lower variance than the PM condition. This indirectly provides some insight into what proprioceptive inputs were contributing to the combined estimates.

It is also important to note that there were clear individual differences, which is not uncommon in studies assessing (optimal) multisensory integration (e.g., Bentvelzen et al. 2009; Werner and Noppeney 2010). Nevertheless, we see that in the majority of cases the multisensory condition reduced the variance in spatial updating (i.e., data points below the horizontal dotted line in Fig. 5b). In general, our results are at odds with other commonly evaluated models of sensory integration including the "winner-take-all" model. Specifically, this model assumes that only the most reliable signal is used, while all others are ignored. Based on this model, it would be expected that performance in the multisensory condition could be equivalent to either of the unisensory conditions, but never better. Clearly, this is not true in the current findings.

\section{Passive movement}

During passive self-motion (PM), mean pointing rates were indistinguishable from veridical performance with no clear indication of biases introduced by the proprioceptive input, which always specified zero velocity. Moreover, the pointing profiles revealed that a characteristic pattern of pointing behavior was generally observed, such that pointing velocity accelerated upon target approach, peaked around target passage, and decelerated after target passage, indicating relatively accurate target-relative updating. However, the patterns of perceived velocity in this condition did not completely overlap with velocity estimates observed in the full-cue condition and appeared to result in underestimates of velocity just before, during, and following the target approach and passage (particularly at the higher velocity, see Fig. 3a). These results are consistent with those from a previous experiment using a continuous pointing task to investigate passive self-motion perception for simple translational movements (Siegle et al. 2009). Moreover, the estimates based mainly on inertial cues appeared relatively unaffected by any conflicting proprioceptive information about self-motion (i.e., lack of any leg movements typically associated with natural forms of movement). We can therefore conclude that the performance in the PM condition is consistent with the hypothesis that being passively moved should not necessarily be considered a conflicting multisensory condition in the same way as walking in place.

\section{Walking in place}

As described earlier, it was predicted that the conflicting zero-movement input from the vestibular system in the WIP condition would not be as readily ignored as the conflicting zero-movement proprioceptive input in the PM condition. Several results support this prediction. For instance, a mandatory integration of these two inputs in the WIP condition should lead to an underestimation of selfmotion. Indeed, mean pointing rates revealed a general underestimation of velocity that was greater at faster velocities. This underestimation is reflected in the vestibular weight calculated from the data $(0.22)$ and could be attributable to the zero-velocity vestibular inputs. This weight is considerably lower than the vestibular weight of 0.62 calculated for the WTS conflict conditions. The difference may be due to the much larger conflict between the proprioceptive walking speed and the zero vestibular input in the WIP condition, compared to the much smaller conflicts in the WTS condition (i.e., subtle gains of 0.7 and 1.4). Indeed, it has been previously reported that relative cue weighting changes with the magnitude of conflict (Gepshtein et al. 2005).

In the WIP condition, the instantaneous pointing rate did not change as a function of egocentric, target-relative position. Instead, participants moved the pointer at a constant rate throughout the entire trial. Consequently, 
proprioceptive information from walking in place did not appear to be sufficient to support the experience of accurate self-motion through space. This could have been due to the reduced perceived self-motion velocity driven by the zero-movement vestibular input (which would tend to flatten the pointing profiles). It could also be due to the additional cognitive processes necessary for transforming the proprioceptive input into imagined self-motion through space. Interestingly, the pattern of pointing behavior observed for the WIP condition in the current study is consistent with observations in a previous study using a similar continuous pointing task where observers purely imagined their target-relative movements through space in the complete absence of any physical movement. Pointing rate profiles in the imagined movement were substantially 'flatter' than those in actual movement conditions (Campos et al. 2009).

There is other evidence, however, to suggest that the proprioceptive information provided when walking in place may not be completely negligible. For instance, Kunz et al. (2009) demonstrated that when comparing responses in an actual time-to-walk task (i.e., chronometric measure), to an imagined time-to-walk task, imagery was facilitated when observers simultaneously performed a behavior consistent with the imagined behavior (i.e., stepping in place) compared to an irrelevant behavior (i.e., waving one's arms). Further, several researchers have also reported that observers often experience a compelling sense of egocentric movement through space during relatively brief periods of blindfolded walking in place, which has been referred to as "vection from walking" (Becker et al. 2002; Bles 1981; Bles and de Wit 1978; Bruggeman 2009). It should be noted that because curvilinear walking was used for the current study, it remains unclear whether such biases in updating would also be observed for other types of movements (e.g., purely translational or purely rotational). Therefore, further investigation into the processes underlying self-motion perception when walking in place in the absence of vision is required.

Overall, the biases in updating performance revealed in the WIP condition mean that it cannot simply be used to make inferences about the sensory noise specific to the proprioceptive system and thus cannot be used to formally test quantitative models such as those based on MLE. Therefore, results from past studies that have used walking in place conditions as a method for evaluating unisensory proprioceptive estimates to study multisensory self-motion perception should be interpreted with caution and alternative methods should be considered. One possibility of eliminating any effects of the conflicting vestibular input in the WIP condition might be to test labyrinthine-defective participants (Glasauer et al. 1994, 2002). However, this would then present the new complication of obtaining within-subject unisensory estimates of sensory noise for the vestibular system.

\section{Conclusion}

In summary, the present study demonstrated that during full-stride curvilinear locomotion, humans use both vestibular and proprioceptive information to estimate their velocity through space and integrate these sources of information in a manner that is consistent with a MLE model. Variance in spatial updating judgments was generally lower in multimodal conditions than in the unimodal ones. Moreover, performance in conflict conditions suggested the use of a weighted average of the available cues. The walking in place condition was shown not to provide an appropriate and independent unisensory estimate of proprioception, raising questions concerning its validity in cue integration research.

Acknowledgments The first two authors contributed equally to this work. We thank Michael Weyel for technical assistance and Dr John Butler for valuable advice on the data analysis. We also thank Dr Jack Loomis for earlier valuable discussions and insights. We gratefully acknowledge the work done by the mechanical and electrical workshops of the Max Planck Institute for Biological Cybernetics in adapting the CTM and making it operational. This work was funded by the European 6th Framework Programme CyberWalk (FP6511092). The writing of this paper by the first author was partially supported by a NSERC grant attributed to Catherine Guastavino (McGill University).

\section{References}

Alais D, Burr D (2004) The ventriloquist effect results from nearoptimal bimodal integration. Curr Biol 14:257-262

Alton F, Baldey L, Caplan S, Morrisey MC (1998) A kinematic comparison between overground and treadmill walking. Clin Biomech 13:434-440

Andre J, Rogers S (2006) Using verbal and blind-walking distance estimates to investigate the two visual systems hypothesis. Percept Psychophys 68:353-361

Angelaki DE, Cullen KE (2008) Vestibular system: the many facets of a multimodal sense. Annu Rev Neurosci 31:125-150

Angelaki DE, Gu Y, DeAngelis GC (2009) Multisensory integration: psychophysics, neurophysiology, and computation. Curr Opin Neurobiol 19:452-458

Bakker NH, Werkoven PJ, Passenier PO (1999) The effects of proprioceptive and visual feedback on geographical orientation in virtual environments. Presence-Teleop Virt 8:36-53

Becker W, Nasios G, Raab S, Jürgens R (2002) Fusion of vestibular and podokinesthetic information during self-turning towards instructed targets. Exp Brain Res 144:458-474

Bentvelzen A, Leung J, Alais D (2009) Discriminating audiovisual speed: optimal integration of speed defaults to probability summation when component reliabilities diverge. Perception 38:966-987

Berthoz A, Israël I, Georges-François P, Grasso R, Tsuzuku T (1995) Spatial memory of body linear displacement: what is being stored? Science 269:95-98 
Bles W (1981) Stepping around: circular vection and Coriolis effects. In: Long J, Baddeley A (eds) Attention and performance IX. Erlbaum, Hillsdale, NJ

Bles W, de Wit G (1978) La sensation de rotation et la march circulaire [sensation of rotation and circular walking]. Aggressologie 19(A):29-30

Bruggeman H, Piuneu VS, Rieser JJ, Pick HL Jr (2009) Biomechanical versus inertial information: stable individual differences in perception of self-rotation. J Exp Psychol Hum Percept Perform 35:1472-1480

Bülthoff HH, Yuille AL (1996) A Bayesian framework for the integration of visual modules. In: McClelland J, Inui T (eds) Attention and performance XVI: information integration in perception and communication. MIT Press, Cambridge

Butler JS, Smith ST, Campos JL, Bülthoff HH (2010) Bayesian integration of visual and vestibular signals for heading. $\mathrm{J}$ Vis 10(11): Article 23. doi:10.1167/10.11.23

Campos JL, Bülthoff HH (in press) Multisensory integration during self-motion in virtual reality. In: Wallace M, Murray M (eds) Frontiers in the neural bases of multisensory processes, Taylor and Francis

Campos JL, Siegle JH, Mohler BJ, Bülthoff HH, Loomis JM (2009) Imagined self-motion differs from perceived self-motion: evidence from a novel continuous pointing method. PLoS ONE 4:e7793. doi:10.1371/journal.pone.0007793

Campos JL, Byrne P, Sun H-J (2010) Body-based cues trump vision when estimating walked distance. Eur J Neurosci 31:1889-1898

Cheng K, Shettleworth SJ, Huttenlocher J, Rieser JJ (2007) Bayesian integration of spatial information. Psychol Bull 133:625-637

Durgin FH, Mikio A, Gallistel CR, Haiken W (2009) The precision of locomotor odometry in humans. Exp Brain Res 193:429-436

Ellard CG, Shaughnessy SC (2003) A comparison of visual and nonvisual sensory inputs to walked distance in a blind-walking task. Perception 32:567-578

Elliott D (1986) Continuous visual information may be important after all: a failure to replicate Thomson. J Exp Psychol Hum Percept Perform 12:388-391

Ernst MO, Banks MS (2002) Humans integrate visual and haptic information in a statistically optimal fashion. Nature 415:429-433

Ernst MO, Bülthoff HH (2004) Merging the senses into a robust percept. Trends Cogn Sci 8:162-169

Faisal AA, Wolpert DM (2009) Near optimal combination of sensory and motor uncertainty in time during a naturalistic perceptionaction task. J Neurophysiol 101:1901-1912

Fetsch CR, Turner AH, DeAngelis GC, Angelaki DE (2009) Dynamic reweighting of visual and vestibular cues during self-motion perception. J Neurosci 29:15601-15612

Fukusima SS, Loomis JM, Da Silva JA (1997) Visual perception of egocentric distance as assessed by triangulation. J Exp Psychol Hum Percept Perform 23:86-100

Gepshtein S, Burge J, Ernst MO, Banks MS (2005) The combination of vision and touch depends on spatial proximity. J Vis 5(11): Article 7. doi:10.1167/5.11.7

Glasauer S, Amorim MA, Vitte E, Berthoz A (1994) Goal-directed linear locomotion in normal and labyrinthine-defective subjects. Exp Brain Res 98:323-335

Glasauer S, Amorim MA, Viaud-Delmon I, Berthoz A (2002) Differential effects of labyrinthine dysfunction on distance and direction during blindfolded walking of a triangular path. Exp Brain Res 145:489-497

Harris LR, Jenkin M, Zikovitz DC (2000) Visual and non-visual cues in the perception of linear self-motion. Exp Brain Res 135:12-21

Israël I, Berthoz A (1989) Contribution of the otoliths to the calculation of linear displacement. J Neurophysiol 62:247-263
Israël I, Grasso R, Georges-Francois P, Tsuzuku T, Berthoz A (1997) Spatial memory and path integration studied by self-driven passive linear displacement. I. Basic properties. J Neurophysiol 77:3180-3192

Jacobs RA (1999) Optimal integration of texture and motion cues to depth. Vis Res 39:3621-3629

Jürgens R, Becker W (2006) Perception of angular displacement without landmarks: evidence for Bayesian fusion of vestibular, optokinetic, podokinesthetic and cognitive information. Exp Brain Res 174:528-543

Knill DC, Saunders JA (2003) Do humans optimally integrate stereo and texture information for judgments of surface slant? Vis Res 43:2539-2558

Körding KP, Wolpert DM (2004) Bayesian integration in sensorimotor learning. Nature 427:244-247

Kunz BR, Creem-Regehr SH, Thompson WB (2009) Evidence for motor simulation in imagined locomotion. J Exp Psychol Hum Percept Perform 35:1458-1471

Lackner JR, DiZio P (2005) Vestibular, proprioceptive, and haptic contributions to spatial orientation. Annu Rev Psychol $56: 115-147$

Loomis JM, Philbeck JW (2008) Measuring perception with spatial updating and action. In: Klatzky RL, Behrmann M, MacWhinney B (eds) Embodiment, egospace and action. Erlbaum, Mahwah, NJ

Loomis JM, DaSilva JA, Fujita N, Fukusima SS (1992) Visual spaceperception and visually-directed action. J Exp Psychol Hum Percept Perform 18:906-921

MacNeilage PR, Banks MS, Berger DR, Bülthoff HH (2007) A Bayesian model of the disambiguation of gravitoinertial force by visual cues. Exp Brain Res 179:263-290

Marlinsky VV (1999) Vestibular and vestibule-proprioceptive perception of motion in the horizontal plane in blindfolded man: I. Estimations of linear displacement. Neuroscience 90:389-394

Mittelstaedt ML, Mittelstaedt H (2001) Idiothetic navigation in humans: estimation of path length. Exp Brain Res 139:318-332

Nardini M, Jones P, Bedford R, Braddick O (2008) Development of cue integration in human navigation. Curr Biol 18:689-693

Rieser JJ, Ashmead DH, Talor CR, Youngquist GA (1990) Visual perception and the guidance of locomotion without vision to previously seen targets. Perception 19:675-689

Rieser JJ, Pick HL Jr, Ashmead DH, Garing AE (1995) Calibration of human locomotion and models of perceptual-motor organization. J Exp Psychol Hum Percept Perform 21:480-497

Riley PO, Paolini G, Della Croce U, Paylo KW, Kerrigan DC (2007) A kinematic and kinetic comparison of overground and treadmill walking in healthy subjects. Gait Posture 26:17-24

Siegle JH, Campos JL, Mohler BJ, Loomis JM, Bülthoff HH (2009) Measurement of instantaneous perceived self-motion using continuous pointing. Exp Brain Res 195:429-444

Souman JL, Frissen I, Sreenivasa MN, Ernst MO (2009) Walking straight into circles. Curr Biol 19:1538-1542

Sperry RW (1950) Neural basis of the spontaneous optokinetic response produced by visual inversion. J Comp Physiol Psychol 43:482-489

Steenhuis RE, Goodale MA (1988) The effects of time and distance on accuracy of target-directed locomotion: does an accurate short-term memory for spatial location exist? J Mot Behav 20:399-415

Sun H-J, Lee AJ, Campos JL, Chan GSW, Zhang DH (2003) Multisensory integration in speed estimation during self-motion. Cyberpsychol Behav 6:509-518

Sun H-J, Campos JL, Chan GSW (2004a) Multisensory integration in the estimation of relative path length. Exp Brain Res 154:246-254 
Sun H-J, Campos JL, Chan GSW, Young M, Ellard C (2004b) The contributions of static visual cues, nonvisual cues, and optic flow in distance estimation. Perception 33:49-65

Thomson JA (1983) Is continuous visual monitoring necessary in visually guided locomotion? J Exp Psychol Hum Percept Perform 9:427-443
Von Holst E, Mittelstaedt H (1950) Das Reafferenzprinzip (Wechselwirkungen zwischen Zentralnervensystem und Peripherie). Naturwissenschaften 37:464-476

Werner S, Noppeney U (2010) Superadditive responses in superior temporal sulcus predict audiovisual benefits in object categorization. Cereb Cortex 20:1829-1842 